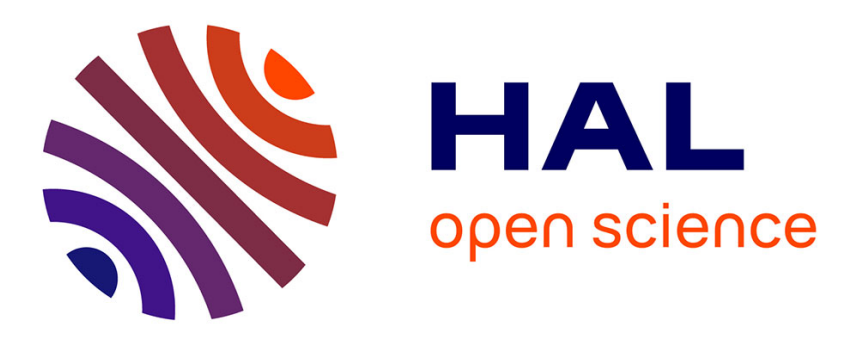

\title{
Advanced lattice Boltzmann scheme for high-Reynolds-number magneto-hydrodynamic flows
}

Alessandro de Rosis, Emmanuel Lévêque, Robert Chahine

\section{To cite this version:}

Alessandro de Rosis, Emmanuel Lévêque, Robert Chahine. Advanced lattice Boltzmann scheme for high-Reynolds-number magneto-hydrodynamic flows. Journal of Turbulence, 2018, 2018, 10.1080/14685248.2018.1461875. hal-02084824

\section{HAL Id: hal-02084824 \\ https://hal.science/hal-02084824}

Submitted on 9 Apr 2019

HAL is a multi-disciplinary open access archive for the deposit and dissemination of scientific research documents, whether they are published or not. The documents may come from teaching and research institutions in France or abroad, or from public or private research centers.
L'archive ouverte pluridisciplinaire HAL, est destinée au dépôt et à la diffusion de documents scientifiques de niveau recherche, publiés ou non, émanant des établissements d'enseignement et de recherche français ou étrangers, des laboratoires publics ou privés. 


\title{
2 Advanced Lattice Boltzmann Scheme for High-Reynolds-number Magneto-Hydrodynamic Flows
}

\author{
A. De Rosis ${ }^{\mathrm{a}, \mathrm{b}}$, Emmanuel Lévêque ${ }^{\mathrm{b}}$, Robert Chahine ${ }^{\mathrm{b}}$ \\ a Department of Biomedical Engineering Technion - Israel Institute of Technology, 32000, \\ Haifa, Israel \\ ${ }^{\text {b} U n i v ~ L y o n ~-~ E c o l e ~ C e n t r a l e ~ d e ~ L y o n ~-~ C N R S ~-~ L a b o r a t o i r e ~ d e ~ M e ́ c a n i q u e ~ d e s ~ F l u i d e s ~ e t ~}$ \\ d'Acoustique, F-69134 Ecully cedex, France
}

\author{
ARTICLE HISTORY \\ Compiled April 9, 2019
}

\begin{abstract}
Is the lattice Boltzmann method suitable to investigate numerically high-Reynolds-number magneto-hydrodynamic (MHD) flows? It is shown that a standard approach based on the Bhatnagar-Gross-Krook (BGK) collision operator rapidly yields unstable simulations as the Reynolds number increases. In order to circumvent this limitation, it is here suggested to address the collision procedure in the space of central moments for the fluid dynamics. Therefore, an hybrid LB scheme is introduced, which couples a central-moment scheme for the velocity with a BGK scheme for the space-and-time evolution of the magnetic field. This method outperforms the standard approach in terms of stability, allowing us to simulate high-Reynolds-number MHD flows with non-unitary Prandtl number while maintaining accuracy and physical consistency.
\end{abstract}

\section{KEYWORDS}

Lattice Boltzmann method, magnetohydrodynamics, turbulence

The use of the lattice Boltzmann (LB) method has become ubiquitous in many areas of computational fluid dynamics, and now represents a consolidate alternative to classical approaches based on the discretization of the incompressible Navier-Stokes equations [1-8]. In short, the flow is inferred from the motion of distributions of fictitious particles streaming and colliding along the links of a regular lattice. The LB method has practical advantages with respect to a continuum-based formulation. In particular, LB dynamics is governed by a first-order partial differential equation in which non-localities and non-linearities are well separated [5]. Conversely, the integration of the Navier-Stokes equations requires the evaluation of first and second-order derivatives, and possibly the application of a non-local Poisson solver to obtain the pressure field. Moreover, the computational complexity of the continuum-based approach becomes rapidly prominent and evident when the fluid dynamics encompasses additional physical features such as magnetic effects. In that case, the particulate nature of the LB approach offers some tangible advantages, as will be demonstrated in this article.

The incompressible Navier-Stokes equations for magnetohydrodynamics (MHD) drive the evolution of an electrically conductive fluid of kinematic viscosity $\nu$ and 
magnetic diffusivity $\eta$ in the form

$$
\begin{aligned}
& \partial_{t} \boldsymbol{u}+(\boldsymbol{u} \cdot \nabla) \boldsymbol{u}=-\frac{\nabla p}{\rho}+\nu \Delta \boldsymbol{u}+\frac{\boldsymbol{j} \times \boldsymbol{b}}{\rho} \\
& \partial_{t} \boldsymbol{b}=\nabla \times(\boldsymbol{u} \times \boldsymbol{b})+\eta \Delta \boldsymbol{b} \\
& \nabla \cdot \boldsymbol{u}=0 \\
& \nabla \cdot \boldsymbol{b}=0
\end{aligned}
$$

where $\rho$ and $\boldsymbol{u}$ are the mass density and velocity of the fluid, respectively. The vector field $\boldsymbol{b}$ denotes the magnetic field and $\boldsymbol{j}=\boldsymbol{\nabla} \times \boldsymbol{b}$ is the electric current. The fluid pressure $p$ stems from the incompressibility constraint $\nabla \cdot \boldsymbol{u}=0$. In comparison with the non-magnetic case, here it is mandatory to integrate a coupled set of non-linear partial differential equations for the velocity and magnetic fields, thus leading to heavy computations.

Our motivation is to explore the possibility to use the LB method to investigate numerically high-Reynolds-number MHD flows with non-unitary Prandtl number. The earliest attempt to build a lattice gas automaton for MHD refers to [9] by Montgomery and Doolen. It is based on a magnetic vector potential formulation. The inclusion of the Lorentz force relies on the computation of a Laplacian operator with the consequent implementation of an additional non-local finite-difference procedure. Later, a purely local lattice gas model has been introduced by Chen et al. [10]. However, this modeling requires to solve a 36-state MHD Cellular Automaton system at each node of a two-dimensional hexagonal lattice, hence leading to a dramatic computational cost. Martinez et al. [11] have managed to reduce the number of states to twelve. In addition, an hybrid scheme coupling the LB approach with finite-difference discretization has been proposed by Succi et al. [12] for two-dimensional MHD, allowing for simulations with a magnetic Prandtl number, defined as the ratio between the kinematic viscosity and the magnetic diffusivity, fixed at unity.

More recently, Dellar has demonstrated that the solution of the aforementioned set of MHD equations may be recovered by solving two coupled LB schemes based on the BGK collision operator [13]. The former involves densities of fictitious particles carrying amount of mass, namely $f_{i}$ in each direction, and accounting for the evolution of the mass density $\rho$ and momentum $\rho \boldsymbol{u}$ of the fluid. The latter involves particles carrying amount of magnetic field, namely $\boldsymbol{g}_{i}$ in each direction, and addressing the dynamics of the magnetic field $\boldsymbol{b}$. This algorithm overcomes the major limitations of the previous efforts. It is purely local, the magnetic Prandtl number $\operatorname{Pr}_{m}$ is not limited at unity and the computational cost is very affordable. This scheme will be considered below and used as a baseline for the development of an improved scheme dedicated to high-Reynolds-number MHD flows.

Following [13], the D2Q9 and D2Q5 lattices are adopted for $f_{i}$ and $\boldsymbol{g}_{i}$, respectively. Here, two-dimensional modeling is considered for the sake of clarity, the extension to three dimensions being straightforward and outlined at the end of the article. The lattice directions are denoted by $\boldsymbol{c}_{i}=\left[\left|c_{i x}\right\rangle,\left|c_{i y}\right\rangle\right]$ with

$$
\begin{aligned}
& \left|c_{i x}\right\rangle=[0,1,0,-1,0,1,-1,-1,1]^{\top}, \\
& \left|c_{i y}\right\rangle=[0,0,1,0,-1,1,1,-1,-1]^{\top},
\end{aligned}
$$

where $|\bullet\rangle$ denotes a column vector and the superscript $\top$ indicates the transpose of a vector. At position $\boldsymbol{x}$ and time $t$, the LB scheme advances the set of distributions in 
a two-step procedure. Namely, a streaming step for fluid particles

$$
f_{i}\left(\boldsymbol{x}+\boldsymbol{c}_{i} \Delta t, t+\Delta t\right)=f_{i}^{\text {coll }}(\boldsymbol{x}, t)
$$

is consecutive to a collision step

$$
f_{i}^{\text {coll }}(\boldsymbol{x}, t)=f_{i}(\boldsymbol{x}, t)-\omega_{\nu}\left[f_{i}(\boldsymbol{x}, t)-f_{i}^{e q}(\boldsymbol{x}, t)\right] .
$$

The so-called BGK approximation refers to this simple form of the collision operator, which expresses as the relaxation with the same rate of all distributions towards absolute equilibrium. Similarly, for the magnetic particles

$$
\boldsymbol{g}_{i}\left(\boldsymbol{x}+\boldsymbol{c}_{i} \Delta t, t+\Delta t\right)=\boldsymbol{g}_{i}^{\text {coll }}(\boldsymbol{x}, t)
$$

with

$$
\boldsymbol{g}_{i}^{\text {coll }}(\boldsymbol{x}, t)=\boldsymbol{g}_{i}(\boldsymbol{x}, t)+\omega_{\eta}\left[\boldsymbol{g}_{i}^{e q}(\boldsymbol{x}, t)-\boldsymbol{g}_{i}(\boldsymbol{x}, t)\right]
$$

Here and henceforth, the index $i$ spans the directions $i=0 \cdots 8$ (D2Q9 lattice) and $i=0 \cdots 4$ (D2Q5 lattice) for the distributions $f_{i}$ and $\boldsymbol{g}_{i}$, respectively. The relaxation frequencies $\omega_{\nu}$ and $\omega_{\eta}$ are related to the kinematic viscosity and magnetic diffusivity of the fluid by

$$
\nu=\left(\frac{1}{\omega_{\nu}}-\frac{1}{2}\right) c_{s}^{2}
$$

and

$$
\eta=\left(\frac{1}{\omega_{\eta}}-\frac{1}{2}\right) \theta^{2}
$$

with $c_{s}^{2}=\theta^{2}=\frac{1}{3}$ in lattice units. In this framework, the variable $c_{s}$ (and $\theta$ ) refers to the characteristic speed of the particles and may be associated to some extent with a lattice sound speed. Since, nearly-incompressible flows are concerned, the related Mach number $\mathrm{Ma}=|\boldsymbol{u}| / c_{s} \ll 1$. Let us recall that in the lattice Boltzmann method, the incompressible limit $\rho=\rho_{0}$ is approached with $\delta \rho / \rho_{0}=O\left(\mathrm{Ma}^{2}\right)$ [14]. The equilibrium distributions are given by

$$
\begin{aligned}
{ }_{93}^{e q} & =w_{i} \rho\left[1+\frac{\boldsymbol{c}_{i} \cdot \boldsymbol{u}}{c_{s}^{2}}+\frac{\left(\boldsymbol{c}_{i} \cdot \boldsymbol{u}\right)^{2}}{2 c_{s}^{4}}-\frac{\boldsymbol{u} \cdot \boldsymbol{u}}{2 c_{s}^{2}}\right] \\
& +\frac{w_{i}}{2 c_{s}^{4}}\left[\frac{1}{2}\left|\boldsymbol{c}_{i}\right|^{2}|\boldsymbol{b}|^{2}-\left(\boldsymbol{c}_{i} \cdot \boldsymbol{b}\right)^{2}\right] \\
{ }_{94} \quad g_{i \beta}^{e q} & =W_{i}\left[b_{\beta}+\frac{c_{\alpha i}}{\theta^{2}}\left(u_{\alpha} b_{\beta}-u_{\beta} b_{\alpha}\right)\right]
\end{aligned}
$$

where $\alpha$ and $\beta$ span the Cartesian coordinates. The weighting factors are $w_{0}=4 / 9$, $w_{1 \ldots 4}=1 / 9, w_{5 \ldots 8}=1 / 36$ for the fluid dynamics, whereas $W_{0}=1 / 3$ and $W_{1 \ldots 4}=1 / 6$ 
for the magnetic field. Finally, the macroscopic fields are inferred locally by

$$
\rho=\sum_{i=0}^{8} f_{i}, \quad \rho \boldsymbol{u}=\sum_{i=0}^{8} f_{i} \boldsymbol{c}_{i}, \quad \boldsymbol{b}=\sum_{i=0}^{4} \boldsymbol{g}_{i} .
$$

Paul Dellar has demonstrated that this LB scheme was compliant with the MHD equations in the continuous limit through a Chapman-Enskog expansion [13].

This original scheme is now tested against the Orszag-Tang vortex problem [13,15]. This test case has become a popular benchmark representative of many features of turbulent MHD flows, such as magnetic reconnection, formation of jets and dynamic alignment. The deterministic initial conditions allows for a direct comparison between several numerical modeling. Precisely, the flow of an electrically conductive fluid develops in a square periodic box of size $L=2 \pi \mathrm{m}$ with the initial fields

$$
\begin{aligned}
\boldsymbol{u}(\boldsymbol{x}, 0) & =u_{0}[-\sin y, \sin x] \\
\boldsymbol{b}(\boldsymbol{x}, 0) & =b_{0}[-\sin y, \sin 2 x]
\end{aligned}
$$

with the reference magnitudes $u_{0}=b_{0}=2$ in physical units. The initial density is uniform with $\rho(\boldsymbol{x}, 0)=1 \mathrm{~kg} / \mathrm{m}^{3}$. In our simulations, each dimension is discretized into $N=1024$ grid points. The grid resolution is therefore $\Delta x=L / N \approx 6 \times 10^{-3} \mathrm{~m}$ and the time step is fixed at $\Delta t=5 \times 10^{-5} \mathrm{~s}$. In lattice units, this yields the reference velocity $u_{0}=2 \times \Delta t / \Delta x \approx 1.6 \times 10^{-2}$ and the Mach number Ma $\equiv u_{0} / c_{s} \approx 3 \times 10^{-2}$. The Reynolds number is defined (in lattice units) as $\operatorname{Re}=u_{0} N / \nu$. Moreover, the magnetic Prandtl number is set to $\operatorname{Pr}_{\mathrm{m}} \equiv \nu / \eta=1$. Five runs have been performed by varying Re between 500 and 5000. In Fig. 1, the time evolution of the maxima of the electric current $j_{\max }(t)=\max _{\boldsymbol{x}}|j(\boldsymbol{x}, t)|$ is displayed. Notice that the current has only one non-zero component $j$. Furthermore, the LB method allows us to compute the current locally and directly from the distributions, thus avoiding the use of additional time-consuming finite-difference operators [16].

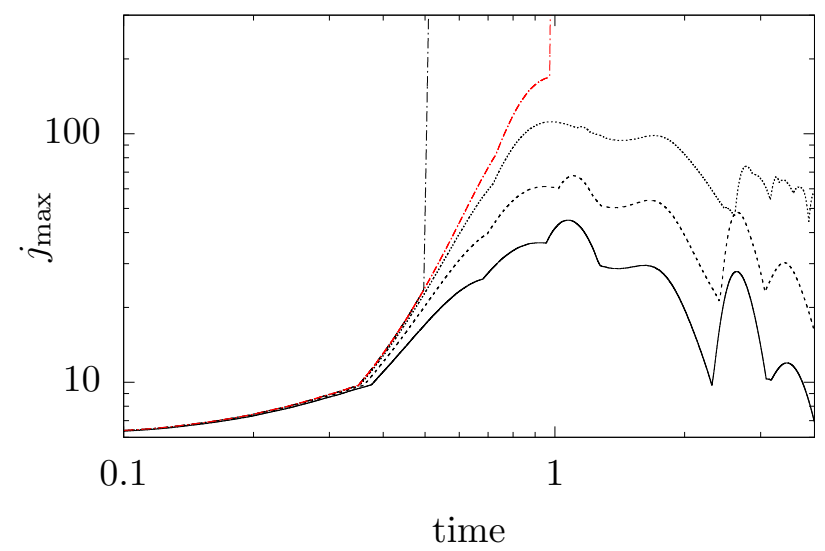

Figure 1. Orszag-Tang vortex problem. LB simulations based on the BGK collision operator [13]. Time evolution of the current maxima at $\mathrm{Re}=500$ (continuous line), 1000 (dashed), 2500 (dotted) and 5000 (dashed-dotted). At the highest Re, an instability occurs at $t \approx 0.52 \mathrm{~s}$. For the same Re, a finer grid consisting of $1536^{2}$ grid points (red dashed-dotted) allows us to extend the life time of the simulation. However, a blow-up eventually occurs at $t \approx 0.99 \mathrm{~s}$. 
Figure 2. Orszag-Tang vortex problem. LB simulations based on the BGK collision operator [13]. Maximal attainable Reynolds number as a function of the magnetic Prandtl number.

The previous observed limitations are related to the very nature of the scheme. Despite its simplicity, effectiveness and large popularity, the BGK collision operator is known to suffer from numerical instabilities when large velocity gradients arise in the flow. Two main factors contribute to this deficiency: The uncontrolled growth of ghost (beyond hydrodynamics) modes [20,21] and the lack of sufficient Galilean invariance [22-28]. By decomposing the collision kernel in a space of raw moments, the multiple-relaxation-time model has proved to increase the stability by properly relaxing high-order moments [29]. However, the lack of Galilean invariance still persists [30]. A possible alleviation of this latter may be addressed by the entropic LBM [31], which was also adopted to investigate MHD turbulence [32]. More recently, a different idea has been proposed by Geier et al. [33] suggesting to relax the moments in a reference frame that moves with the fluid. This can be simply achieved by shifting the lattice velocities by the local fluid velocity, that is

$$
\left|\bar{c}_{i x}\right\rangle=\left|c_{i x}-u_{x}\right\rangle \quad \text { and } \quad\left|\bar{c}_{i y}\right\rangle=\left|c_{i y}-u_{y}\right\rangle
$$

In this case, the involved quantities are called central moments (CMs). This method 
is also referred to as "cascaded" LB scheme, since the post-collision state of any central moment depends only on moments of lower order thus generating a pyramidal hierarchical structure [34-38]. The numerical implementation of the cascaded LB scheme is known to be cumbersome. Nevertheless, some recent attempts have demonstrated that a simplified version of the CMs-based scheme (in a non-orthogonal basis) may be derived, entailing easier implementations [39-41]. This approach is here applied in the context of high-Reynolds-number MHD flows for the fluid particles.

By introducing the basis

$$
\overline{\mathbf{T}}=\left[\overline{\mathbf{t}}_{0}, \ldots, \overline{\mathbf{t}}_{i}, \ldots, \overline{\mathbf{t}}_{8}\right],
$$

with

$$
\begin{array}{rlrl}
\overline{\mathbf{t}}_{0} & =\mid 1,1,1,1,1,1,1,1,1]^{\top}, \\
\overline{\mathbf{t}}_{1} & =\left|\bar{c}_{i x}\right\rangle, & \overline{\mathbf{t}}_{2}=\left|\bar{c}_{i y}\right\rangle, \\
\overline{\mathbf{t}}_{3}=\left|\bar{c}_{i x}^{2}+\bar{c}_{i y}^{2}\right\rangle, & \overline{\mathbf{t}}_{4}=\left|\bar{c}_{i x}^{2}-\bar{c}_{i y}^{2}\right\rangle, \\
\overline{\mathbf{t}}_{5}=\left|\bar{c}_{i x} \bar{c}_{i y}\right\rangle, & \overline{\mathbf{t}}_{6}=\left|\bar{c}_{i x}^{2} \bar{c}_{i y}\right\rangle, \\
\overline{\mathbf{t}}_{7}=\left|\bar{c}_{i x} \bar{c}_{i y}^{2}\right\rangle, & \overline{\mathbf{t}}_{8}=\left|\bar{c}_{i x}^{2} \bar{c}_{i y}^{2}\right\rangle,
\end{array}
$$

a suitable set of central moments is represented by

$$
\left|k_{i}\right\rangle=\left[k_{0}, \ldots, k_{i}, \ldots, k_{8}\right]^{\top},
$$

with

$$
\left|k_{i}\right\rangle=\overline{\mathbf{T}}^{\top}\left|f_{i}\right\rangle
$$

and $\left|f_{i}\right\rangle=\left[f_{0}, f_{1}, f_{2}, f_{3}, f_{4}, f_{5}, f_{6}, f_{7}, f_{8}\right]^{\top}$. Each moment relaxes to an equilibrium state, $k_{i}^{e q}$, defined by replacing $f_{i}$ with $f_{i}^{e q}$ in Eq. (10). The resulting expressions of the equilibrium CMs are

.

One can immediately notice the presence of some terms accounting for the magnetic field, stemming from the second term at the right-hand side of Eq. (1). The collision 
operator reads

$$
k_{i}^{\star}=k_{i}+\omega_{i}\left(k_{i}^{e q}-k_{i}\right) \quad \text { with } i=3 \ldots 8,
$$

where $\omega_{i}$ is the relaxation frequency associated with the moment $k_{i}$. The superscript $\star$ refers to post-collision values. To be compliant with the MHD equations in the continuous limit, only the frequencies related to $k_{4}$ and $k_{5}$ need to be specified as a function of the fluid kinematic viscosity. Specifically, $\nu=\left(\frac{1}{\omega_{\nu}}-\frac{1}{2}\right) c_{s}^{2}$ with $\omega_{4}=$ $\omega_{5}=\omega_{\nu}$. The frequency $\omega_{3}$ is related to the bulk viscosity, whereas $\omega_{6}, \omega_{7}$ and $\omega_{8}$ are associated to higher-order ghost moments and can be set equal to unity, i.e. these moments are fixed at their equilibrium value after the collision step. Let us note that $k_{0}, k_{1}$ and $k_{2}$ are invariant with respect to the collision and are not involved in the collision step.

The post-collision central moments eventually yield the post-collision populations by inverting the mapping Eq. (10):

$$
\left|f_{i}^{\star}\right\rangle=\left(\overline{\mathbf{T}}^{\top}\right)^{-1}\left|k_{i}^{\star}\right\rangle
$$

with $\left|k_{i}^{\star}\right\rangle=\left[\rho, 0,0, k_{3}^{\star}, \ldots, k_{8}^{\star}\right]^{\top}$ and $\left|f_{i}^{\star}\right\rangle=\left[f_{0}^{\star}, \ldots, f_{8}^{\star}\right]^{\top}$. The collision step is followed up with a streaming of the populations towards their neighboring nodes on the lattice ${ }^{1}$. Note that this scheme only involves the evolution of the $f_{i}$ 's for the fluid particles. The evolution of the magnetic distributions $\boldsymbol{g}_{i}$ relies on the standard BGK collision operator, hence resulting in an hybrid scheme that combines CMs and multi-time relaxation for the fluid density and momentum, and single-time relaxation for the magnetic field.

The tests at variable Re and fixed $\mathrm{Pr}_{\mathrm{m}}=1$ previously performed with the BGK collision operator (see Fig. 1) are now reproduced by implementing our hybrid LB scheme. In Fig. 3, the current maxima are displayed.

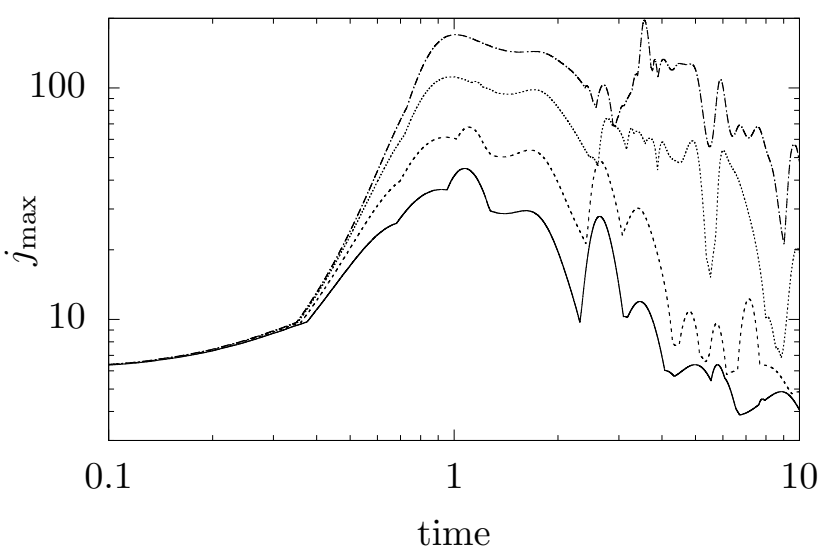

Figure 3. Orszag-Tang vortex problem. Hybrid LB simulation: Time evolution of the current maxima at $\operatorname{Re}=500$ (continuous line), 1000 (dashed), 2500 (dotted) and 5000 (dashed-dotted).

It can be immediately appreciated that the stability is drastically enhanced. In

\footnotetext{
${ }^{1}$ In the Supplementary Material, a script CentralMoments_MHD.m is attached allowing the reader to derive the entire formulation.
} 
practice, a grid consisting of $1024^{2}$ points now allows us to overcome the limit $t \approx$ $0.99 \mathrm{~s}$, for which a finer space-and-time resolution had led to a blow-up with the BGK scheme. After an exponential growth, a faster self-similar increase is experienced with $j_{\max } \sim t^{3}$. It should be noted that this drastic change is slightly anticipated for larger Re. After reaching the peak value, the curves decrease with large oscillations. The decay is less prominent at high Re. These LB results are fully consistent with the previous reports in $[42,43]$.

Accuracy is now examined by straightforward comparisons with the pseudo-spectral data reported in the seminal Orszag-Tang's paper [15]. The time evolution of the kinetic, magnetic and total energies of the flow is well captured in Fig. 4(a). The growth of the magnetic energy and the evolution of the dissipation rate are shown for various values of $\nu=\eta$ in Fig. 4(b) and Fig. 4(c). Overall, a good agreement can be appreciated between the present results and findings in [15] for the global behavior (or $L_{2}$-norm) of the flow and its derivatives. To further validate the accuracy of our numerical scheme, the $L_{\infty}$-norm of the vorticity and electric current is compared in Table 1 to those obtained in a high-resolution pseudo-spectral simulation at $\operatorname{Re} \approx 628$ at $\operatorname{Pr}_{\mathrm{m}}=1$ [13]. The current and vorticity maxima are registered at time instants $t=0.5 \mathrm{~s}$ and $t=1 \mathrm{~s}$. The latter is evaluated as $\zeta_{\max }=\max _{\boldsymbol{x}}|\zeta(\boldsymbol{x})|$ with $\zeta=\boldsymbol{\nabla} \times \boldsymbol{u}$ being the vorticity. The relative discrepancy (in percents) with the pseudo-spectral values is denoted by err. It appears that the relative error slightly increases in time, which may be related to the rise of very large gradients both in the magnetic and velocity fields, as time advances. However, the agreement remains very satisfactory.

\begin{tabular}{c|c|c|c|c} 
& $t(\mathrm{~s})$ & {$[13]$} & Present & $\operatorname{err}(\%)$ \\
\hline \multirow{2}{*}{$j_{\max }$} & 0.5 & 18.24 & 18.24 & 0 \\
& 1 & 46.59 & 46.65 & 0.13 \\
\hline \multirow{2}{*}{$\zeta_{\max }$} & 0.5 & 6.758 & 6.756 & 0.03 \\
& 1 & 14.20 & 14.18 & 0.14
\end{tabular}

Table 1. Orszag-Tang vortex problem at $\operatorname{Re} \approx 628\left(\operatorname{Pr}_{\mathrm{m}}=1\right)$. Reference spectral values from [13] and our results for the peak values of the electric current, $j_{\max }$, and vorticity, $\zeta_{\max }$, at two representative time instants.

The distribution of the kinetic and magnetic energies among resolved wavenumbers is represented by the power-density spectra $E(k)$. This latter is defined as the amount of energy in the shell $k \leq\left|\boldsymbol{k}^{\prime}\right|<k+1$. A direct comparison has been made with the spectra reported by Politano et al. in [44] for the same Orszag-Tang vortex problem solved by a pseudo-spectral method. The Reynolds number is sufficient high to ensure a fully developed turbulence over a broad range of (inertial) scales. Specifically, $\mathrm{Re} \simeq 12600$ with the kinematic viscosity and magnetic diffusivity $\nu=\mu=10^{-3}$ in physical units. For a fair comparison, the grid size is the same, namely, $1024 \times 1024$ in both simulations. The existence of an inertial range with a power-law scaling is visible for both fields in Fig. 5(a) and Fig. 5(b) together with a rapid decline at large wavenumbers due to dissipation. The LB spectra agree fairly well with the pseudo-spectral results in the inertial range, especially for the magnetic field. However, some obvious discrepancies are observed in the dissipation range. One may argue that the LB simulation, which is only second-order accurate in space, under-resolves the gradients of velocity and magnetic field, and therefore underestimates the dissipation rate. This results in an build-up of energy at large wavenumbers. The power-density spectra of kinetic and magnetic enstrophies are considered in Fig. 5(c). The enstrophy power-density spectrum is defined as $\Omega(k)=k^{2} E(k)$ and thus enhances gradient statistics. As previously, we observe that the spectral properties of both velocity and 
magnetic gradients are well resolved in the inertial range but suffers from numerical errors at the largest wavenumbers where dissipation prevails. As already mentioned in the literature, the dominance of the magnetic over the kinetic enstrophy is observed at all wavenumbers.

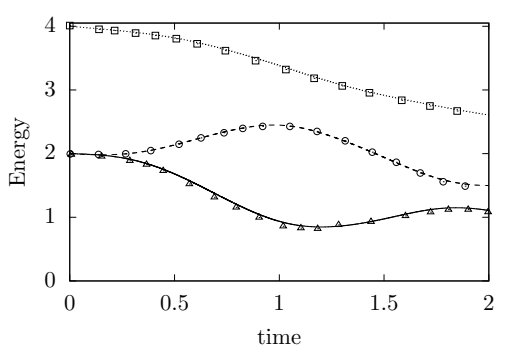

(a)

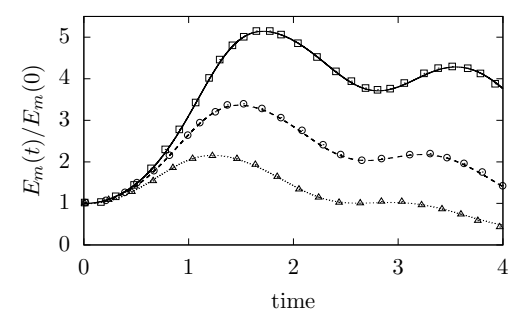

(b)

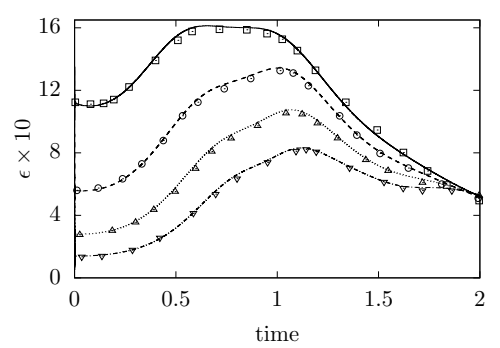

(c)

Figure 4. Orszag-Tang vortex problem in two dimensions. The symbols denote pseudo-spectral data whereas lines correspond to the present LB results. (a) Time evolution of the kinetic (solid line, triangles), magnetic (dashed line, circles) and total (dotted line, squares) energies of the flow with initial condition $u_{0}=b_{0}=1$ and $\nu=\eta=0.02$ in physical units. (b) Time evolution of the magnetic energy $E_{m}(t) / E_{m}(0)$ with a seed magnetic field. The initial condition satisfies $u_{0}=1$ and $\left(b_{0} / u_{0}\right)^{2}=10^{-4}$ with $\nu=\eta=0.01$ (solid line, squares), $\nu=\eta=0.02$ (dashed line, circles) and $\nu=\eta=0.04$ (dotted line, triangles). (c) Time evolution of the dissipation rate with initial conditions $u_{0}=b_{0}=1$ and $\nu=\mu=0.08$ (continuous line, squares), $\nu=\mu=0.04$ (dashed line, circles), $\nu=\mu=0.02$ (dotted line, triangles), $\nu=\mu=0.01$ (dashed-dotted line, inverted triangles)

The LB scheme integrates the fluid dynamics at a mesoscopic level by dealing with populations of particles moving in the different lattice-directions at the speed of sound. It is therefore important to check that within a subvolume of the flow, the macroscopic energy budget is consistently recovered when averaging over all populations of particles. At the macroscopic level, the total energy evolves as

$$
\frac{\partial E}{\partial t}=-\nabla \cdot\left(\left(\frac{1}{2} \rho|\boldsymbol{u}|^{2}+p\right) \boldsymbol{u}-(\boldsymbol{u} \times \boldsymbol{b}) \times \boldsymbol{b}\right)+\rho \nu \boldsymbol{u} \cdot \Delta \boldsymbol{u}+\eta \boldsymbol{b} \cdot \Delta \boldsymbol{b}
$$

with $E=\frac{1}{2} \rho|\boldsymbol{u}|^{2}+\frac{1}{2}|\boldsymbol{b}|^{2}$ being the sum of the kinetic and magnetic energies. By integrating this equation over a subvolume, it is expected that the variation of energy in the subvolume results from the fluxes across the boundaries of the subvolume, stemming from the divergence term in Eq. (14), and the sink of energy due to the kinetic and magnetic dissipations (last two terms). This energy budget is clearly well verified in our LB simualtion, as shown in Fig. 6.

The capability to handle non-unitary magnetic Prandtl numbers is now examined. Therefore, the previous simulation is repeated with $\operatorname{Pr}_{\mathrm{m}}=0.5,1,2$. This is achieved by varying the magnetic diffusivity. In Fig. 7, the space-averaged magnetic energy 


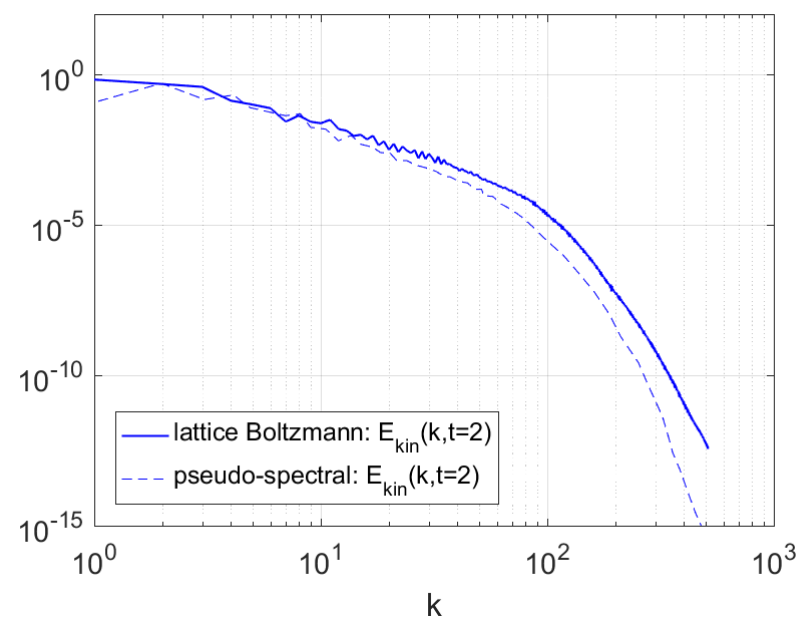

(a)

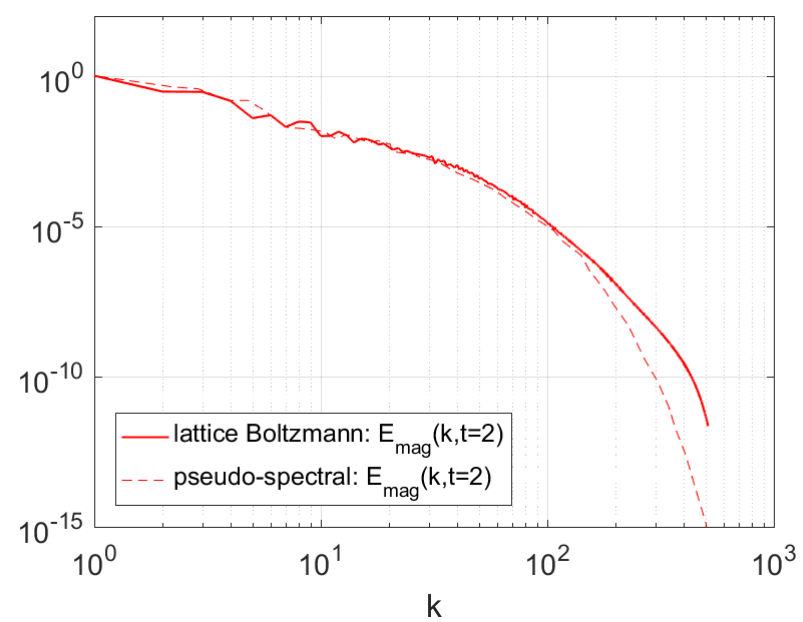

(b)

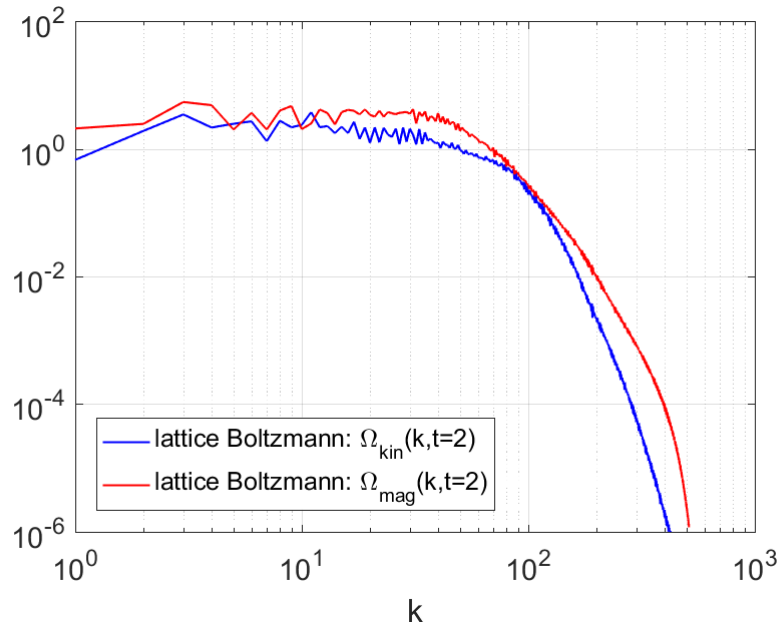

(c)

Figure 5. Comparisons between LB and pseudo-spectral data for the Orszag-Tang vortex problem in two dimensions. The grid size is $1024 \times 1024$ in both simulations. The Reynolds number is $R e \simeq 12600$ and the magnetic Prandtl number is $\operatorname{Pr}_{\mathrm{m}}=1$. (a) kinetic power-density spectra (b) magnetic power-density spectra (c) kinetic and magnetic enstrophy power-density speqto (from LB simulation only). 


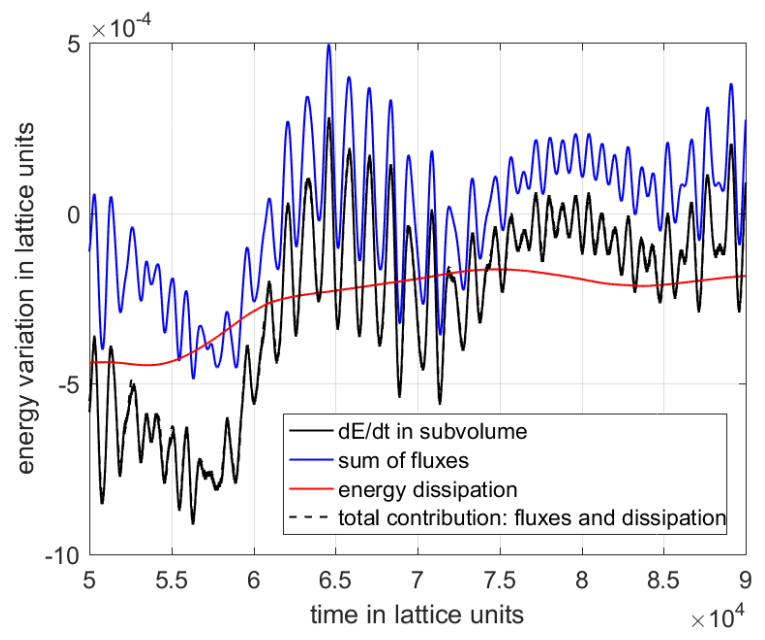

Figure 6. Orszag-Tang vortex problem at $R e=5000$. The time variation of the total energy in a subvolume of size $L / 2 \times L / 2$ is consistently related to the total contribution of the fluxes across the boundaries of the subvolume and the energy dissipation within the subvolume.

$E_{m}=\frac{1}{N^{2}} \sum_{\boldsymbol{x}}|b(\boldsymbol{x})|^{2}$, kinetic energy $E_{k}=\frac{1}{N^{2}} \sum_{\boldsymbol{x}}|u(\boldsymbol{x})|^{2}$ and total energy $E=E_{m}+E_{k}$ are plotted as a function of time. The adoption of a constant $\nu$ explains the substantial

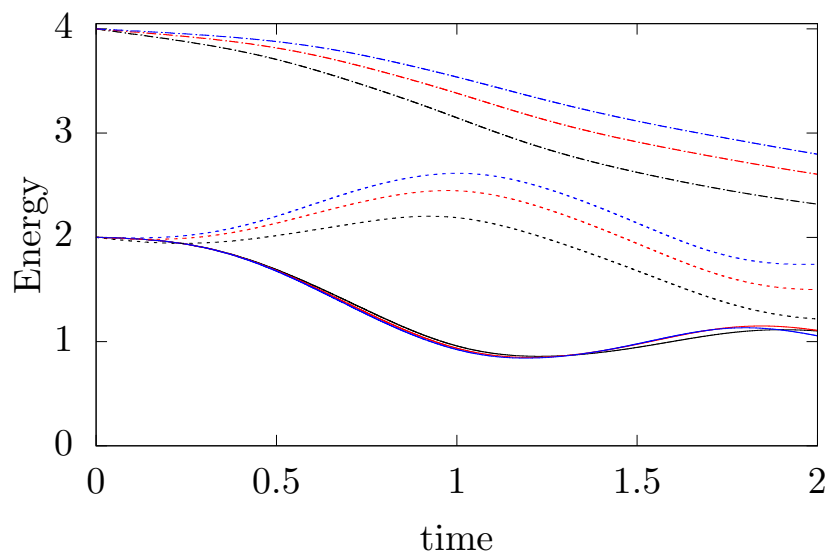

Figure 7. Orszag-Tang vortex problem at $\mathrm{Re} \approx 628$. Time evolution of the space-average kinetic (continuous lines), magnetic (dotted lines) and total (dashed-dotted lines) energies with $\operatorname{Pr}_{\mathrm{m}}=0.5$ (black), 1 (red) and 2 (blue). The kinetic energy does not experience a large influence. Conversely, the magnetic energy increases with $\operatorname{Pr}_{\mathrm{m}}$ due to the reduction of the magnetic diffusivity $\eta$.

insensitivity of the kinetic energy to the variation of $\operatorname{Pr}_{\mathrm{m}}$. Conversely, the magnetic energy, and the total energy as a consequence, undergoes large variations. In particular, $E_{m}$ increases with $\operatorname{Pr}_{m}$ as the magnetic diffusivity reduces. Independently from the magnetic Prandtl number, a significant transfer of energy operates between the magnetic field and the flow, which is fully consistent with the original observations reported by Orszag and Tang in [15].

Further insights are available in Fig. 8(a), where the space-averaged magnetic enstrophy is reported as a function of time. This quantity is computed as $\mathcal{E}_{m}=$ $\frac{1}{N^{2}} \sum_{\boldsymbol{x}} j(\boldsymbol{x})^{2}$. After reaching a maximum at $t \approx 1.2 \mathrm{~s}$, the curves corresponding to $\operatorname{Re}=500$ and $\operatorname{Re}=1000$ rapidly decay as $\sim t^{-2}$ with oscillations reflecting those experienced for the current maxima. As the Reynolds number increases, a plateau 
Fig. 8(b) shows the overall dissipation rate $\epsilon=\nu \mathcal{E}_{k}+\eta \mathcal{E}_{m}$, where the kinetic enstrophy

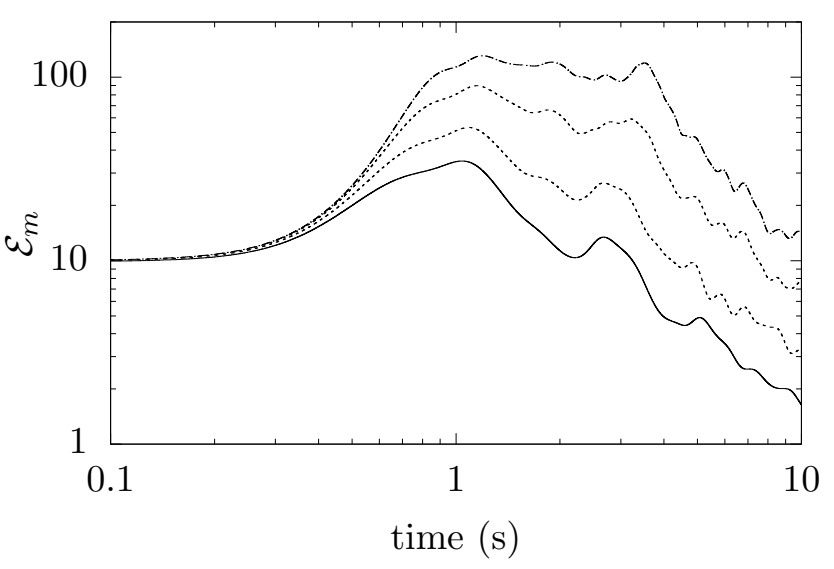

(a)

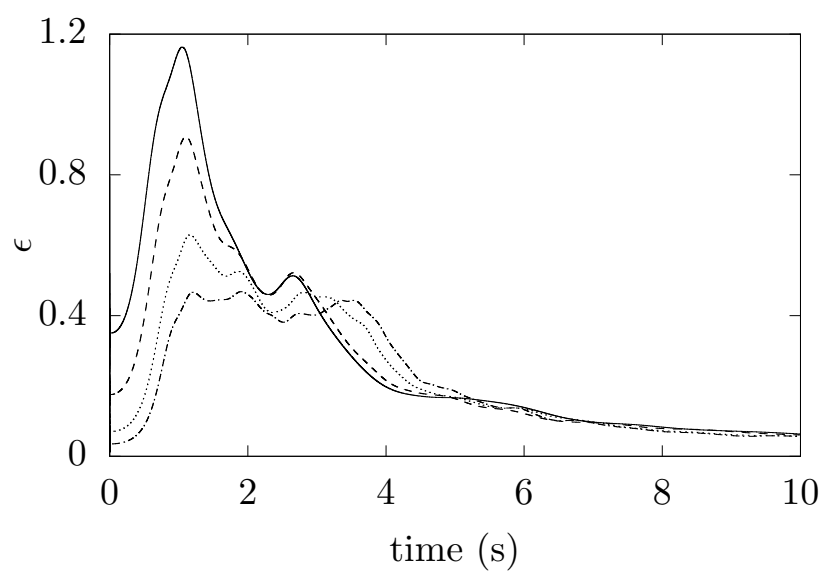

(b) is observed after the initial growth. The local maximum at $t \approx 3.5 \mathrm{~s}$ for the flow at $\operatorname{Re}=5000$ justifies the peak of $j_{\max }$ at that time instant. Eventually, all the

Figure 8. Orszag-Tang vortex problem. (a) Time evolution of the magnetic enstrophy at Re $=500$ (continuous line), 1000 (dashed), 2500 (dotted) and 5000 (dashed-dotted). (b) Time evolution of the overall dissipation rate.

is $\mathcal{E}_{k}=\frac{1}{N^{2}} \sum_{\boldsymbol{x}} \zeta(\boldsymbol{x})^{2}, \zeta=\boldsymbol{\nabla} \times \boldsymbol{u}$ being the vorticity. In the earliest stage, the dissipation increases as Re decreases, highlighting a strong incidence of fluid and magnetic diffusivities. The dissipation rate exhibits a peak at the beginning of the flow. This initial increase is related to the development of small-scale structures in the velocity and magnetic fields. After this transient stage, $\epsilon$ reaches a plateau with a common value for the highest Reynolds numbers. This feature supports Pouquet's hypothesis that the dissipation rate should converge towards a finite non-zero limit as $\nu=\eta \rightarrow 0$ in the developed regime [45]. This plateau is very apparent for the flow at $\operatorname{Re}=5000$. In agreement with [15], this suggests that a flow singularity with $\zeta \rightarrow \infty$, i.e. flow structures of arbitrarily small size may occur at a finite time when $\operatorname{Re} \rightarrow \infty$.

In Fig. 9, the contour plot of the electric current at salient time instants give a better insight of the dynamics of the magnetic field. At $t=1 \mathrm{~s}$, the field exhibits few folds. A straight current sheet passes through the center of the domain, where the maximum is located. This central current sheet goes unstable and very thin structures develop in the flow. At $t=5 \mathrm{~s}$, folds seem to surround two big oculi separated by the 
central sheet, which it is now stabilized. As the time advances, these two big zones are
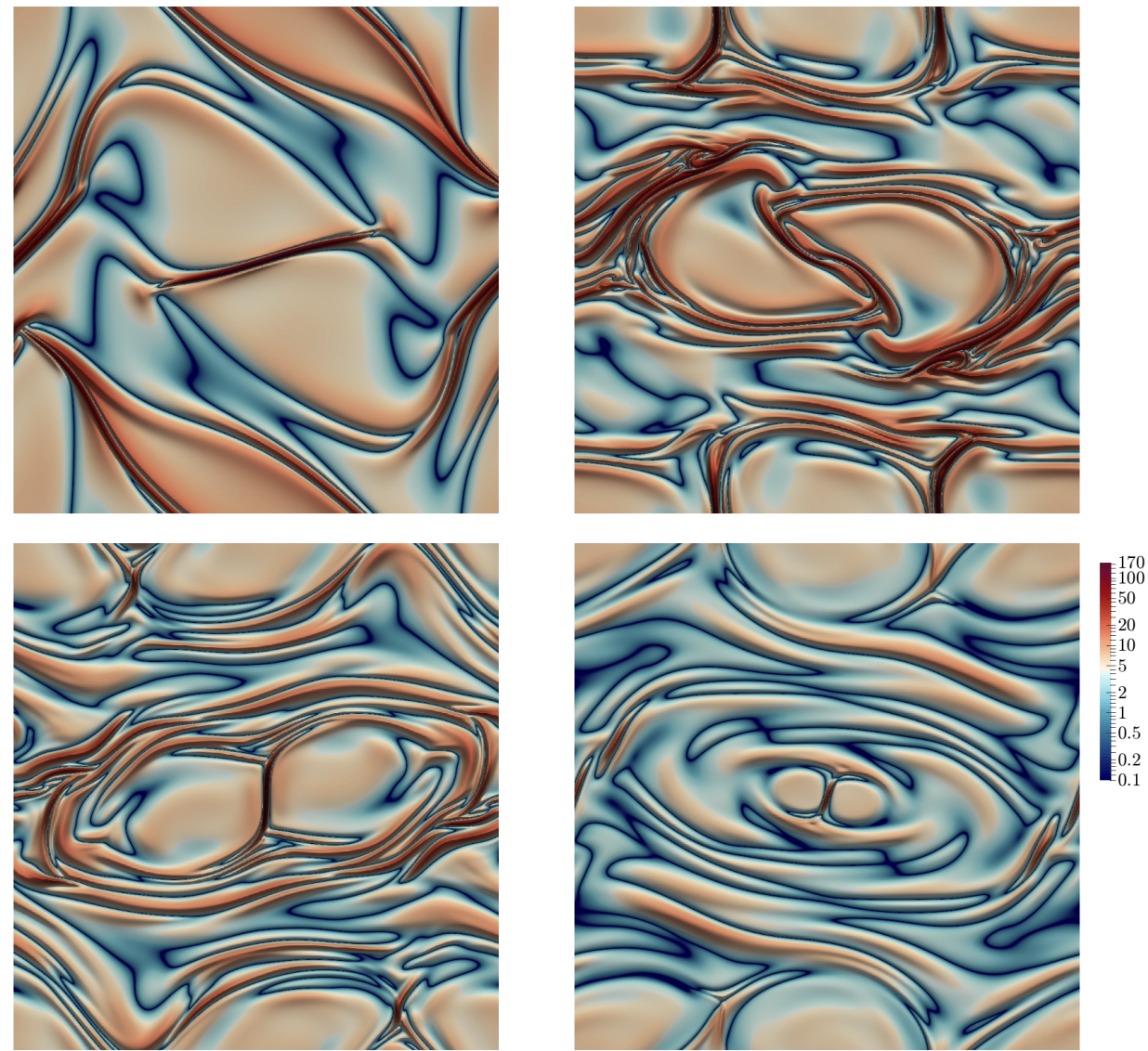

Figure 9. Evolution of the electric current at $\mathrm{Re}=5000$ at salient time instants, i.e. $t=1 \mathrm{~s}$ (top left), $3.5 \mathrm{~s}$ (top right), $5 \mathrm{~s}$ (bottom left) and $9 \mathrm{~s}$ (bottom right). The maximal current is initially located in the central current sheet. The current field undergoes instabilities and many folds arise. Eventually, the central sheet becomes stable again and small-scale structures disappear progressively.

progressively damped by the diffusivities. An Alfenization of the flow, i.e. $\boldsymbol{u}= \pm \boldsymbol{b}$, is expected in the region of high concentration of folds [42]. A quantitative assessment of this effect can be obtained by evaluating the correlation coefficient between the velocity and magnetic fields as $r=\frac{2 \boldsymbol{u} \cdot \boldsymbol{b}}{u^{2}+b^{2}}$. The map of its absolute value is plotted at $t=3.5 \mathrm{~s}$ in Fig. 10. We observe that the correlation is more marked in the vicinity of the current sheets, whereas $\boldsymbol{u}$ and $\boldsymbol{b}$ remain mostly uncorrelated in the rest of the domain. This effect is very well captured by our LB simulation. Finally, our proposed scheme shows an impressive stability even for low values of the magnetic Prandtl number. In fact, we are able to simulate scenarios with vanishing $\operatorname{Pr}_{\mathrm{m}}$ (as $\nu \rightarrow 0$ ) without experiencing the limitations stemming from the adoption of the BGK model.

The possibility to extend the formulation of our hybrid LB scheme to three dimensions is now outlined. In that case, the D3Q27 and D3Q7 lattices should be used for the distributions $f_{i}$ and $\boldsymbol{g}_{i}$, respectively. For the magnetic field, the LB scheme shall still rely on the BGK collision operator with $\theta^{2}=1 / 4$ and the weights $W_{i}$ related to the D3Q7 lattice. For the velocity field, the scheme should be handled according to the CMs-based scheme recently introduced in [40]. In short, it consists of building the matrix $\overline{\mathbf{T}}$ in the D3Q27 velocity space and to compute pre-collision, equilibrium 


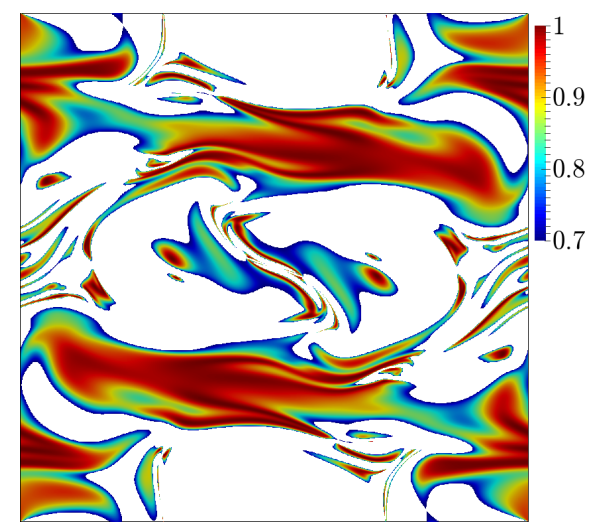

Figure 10. Absolute value of the correlation coefficient $r$ at time instant $t=3.5 \mathrm{~s}$. The flow shows strong correlation in the proximity of current sheets.

and post-collision CMs accordingly. The overall construction of the algorithm remains unaltered.

In conclusion, we have demonstrated the feasibility of the LB method to investigate high-Reynolds MHD flows at non-unitary Prandtl number with an hybrid scheme. Specifically, it is fruitful to decompose the collision stage entering in the dynamics of the fluid velocity in the space of central moments in order to overcome the stability limitations affecting the BGK scheme. In two-dimensions, we have shown that this hybrid scheme enables to reproduce very accurately the key features of the Orszag-Tang vortex problem. Its implementation is not awkward and the generalization to three dimensions is rather straightforward. Eventually, it is worth mentioning that it is here shown that decomposing the collision operator in the space of central moments and relaxing non-hydrodynamical moments to statistical equilibrium provides some tangible advantages from a numerical viewpoint without notably deprecating the physical consistency of the scheme. Alternatively, some variants of the standard LB approach based on some enriched collision operator accounting for high-order statistical moments have recently been proved to better handle strong departure from equilibrium in hydrodynamic and thermodynamic behaviors in various complex flows [46-48]. It would be interesting to compare our rather rustic approach with such more elaborated (but more demanding computationally) mesoscopic modeling.

\section{Supplementary material}

A script is provided in the Supplementary Material (D2Q9_CentralMoments_MHD.m) allowing the reader to perform all the symbolic manipulations to obtain the proposed scheme.

This work has been carried out within the framework of the "Programme Avenir Lyon Saint-Etienne" at the Université de Lyon (ANR-11-IDEX-0007) under the Program "Investissements d'Avenir" operated by the French National Research Agency. The research leading to these results has received funding from the People Programme (Marie Curie Actions) of the European Union's Seventh Framework Programme (FP7/2007-2013) under REA grant agreement $\mathrm{n}^{\circ}$ PCOFUND-GA-2013-609102, through the PRESTIGE programme coordinated by Campus France. 
This article is based upon work from COST Action MP1305, supported by COST (European Cooperation in Science and Technology). The authors are grateful to W.J.T. Bos and N. Plihon for useful hints about magneto-hydrodynamic turbulence.

\section{References}

[1] McNamara GR, Zanetti G. Use of the boltzmann equation to simulate lattice-gas automata. Phys Rev Lett. 1988;61(20):2332.

[2] Higuera F, Succi S, Benzi R. Lattice gas dynamics with enhanced collisions. Europhys Lett. 1989;9(4):345-349.

[3] Benzi R, Succi S, Vergassola M. The lattice Boltzmann equation: Theory and applications. Phys Rep. 1992;222(3):145-197.

[4] Chen S, Doolen G. Lattice Boltzmann method for fluid flows. Annu Rev Fluid Mech. 1998;30(1):329-364.

[5] Succi S. The lattice Boltzmann equation for fluid dynamics and beyond. Clarendon; 2001.

[6] Chen H, Kandasamy S, Orszag S, et al. Extended boltzmann kinetic equation for turbulent flows. Science. 2003;301(5633):633-636.

[7] Succi S. Lattice Boltzmann 2038. Europhys Lett. 2015;109(5):50001-50007.

[8] Succi S. Chimaera simulation of complex states of flowing matter. Phil Trans R Soc A. 2016;374(2080):20160151.

[9] Montgomery D, Doolen GD. Magnetohydrodynamic cellular automata. Phys Lett A. 1987; 120(5):229-231.

[10] Chen H, Matthaeus W, Klein L. An analytic theory and formulation of a local magnetohydrodynamic lattice gas model. Phys Fluids. 1988;31(6):1439-1455.

[11] Martínez DO, Chen S, Matthaeus WH. Lattice boltzmann magnetohydrodynamics. Phys Plasmas. 1994;1(6):1850-1867.

[12] Succi S, Vergassola M, Benzi R. Lattice boltzmann scheme for two-dimensional magnetohydrodynamics. Phys Rev A. 1991 Apr;43:4521-4524. Available from: http: //link.aps.org/doi/10.1103/PhysRevA.43.4521.

[13] Dellar PJ. Lattice kinetic schemes for magnetohydrodynamics. J Comput Phys. 2002; 179(1):95-126.

[14] Dellar PJ. Incompressible limits of lattice boltzmann equations using multiple relaxation times. J Comput Phys. 2003;190(2):351-370.

[15] Orszag SA, Tang CM. Small-scale structure of two-dimensional magnetohydrodynamic turbulence. J Fluid Mech. 1979;90(01):129-143.

[16] Pattison M, Premnath K, Morley N, et al. Progress in lattice boltzmann methods for magnetohydrodynamic flows relevant to fusion applications. Fusion Eng Des. 2008; 83(4):557-572.

[17] Frisch U, Pouquet A, Sulem PL, et al. The dynamics of two-dimensional ideal mhd. J Mec Theor et Appl. 1983;1:191-216.

[18] Klapper I, Rado A, Tabor M. A lagrangian study of dynamics and singularity formation at magnetic null points in ideal three-dimensional magnetohydrodynamics. Phys Plasmas. 1996;3(11):4281-4283.

[19] Grauer R, Marliani C. Current-sheet formation in 3d ideal incompressible magnetohydrodynamics. Phys Rev Lett. 2000;84(21):4850.

[20] Chen H, Teixeira C, Molvig K. Digital physics approach to computational fluid dynamics: Some basic theoretical features. Int J Mod Phys C. 1997;08(04):675-684.

[21] Latt J, Chopard B. Lattice boltzmann method with regularized pre-collision distribution functions. Math Comput Simulat. 2006;72(2-6):165 - 168.

[22] Qian YH, Orszag SA. Lattice bgk models for the navier-stokes equation: Nonlinear deviation in compressible regimes. Europhys Lett. 1993;21(3):255.

[23] Nie XB, Shan X, Chen H. Galilean invariance of lattice boltzmann models. EPL. 2008; 
81(3):34005.

[24] Chen Y, Ohashi H, Akiyama M. Thermal lattice bhatnagar-gross-krook model without nonlinear deviations in macrodynamic equations. Phys Rev E. 1994 Oct;50:2776-2783.

[25] Qian YH, Zhou Y. Complete galilean-invariant lattice bgk models for the navier-stokes equation. Europhys Lett. 1998;42(4):359.

[26] Házi G, Kávrán P. On the cubic velocity deviations in lattice boltzmann methods. J Phys A Math Gen. 2006;39(12):3127.

[27] Keating B, Vahala G, Yepez J, et al. Entropic lattice boltzmann representations required to recover navier-stokes flows. Phys Rev E. 2007;75(3):036712.

[28] Dellar PJ. Lattice boltzmann algorithms without cubic defects in galilean invariance on standard lattices. J Comput Phys. 2014;259:270 - 283.

[29] d'Humières D. Multiple-relaxation-time lattice Boltzmann models in three dimensions. Philos T R Soc A. 2002;360(1792):437-451.

[30] Chen H, Gopalakrishnan P, Zhang R. Recovery of galilean invariance in thermal lattice boltzmann models for arbitrary prandtl number. Int J Mod Phys C. 2014;25(10):1450046.

[31] Boghosian BM, Love PJ, Coveney PV, et al. Galilean-invariant lattice-boltzmann models with h theorem. Phys Rev E. 2003;68(2):025103.

[32] Vahala G, Keating B, Soe M, et al. Mhd turbulence studies using lattice boltzmann algorithms. Commun Comput Phys. 2008;4:624-646.

[33] Geier M, Greiner A, Korvink J. Cascaded digital lattice Boltzmann automata for high Reynolds number flow. Phys Rev E. 2006;73(6):066705.

[34] Geier M, Greiner A, Korvink J. Properties of the cascaded lattice Boltzmann automaton. Int J Mod Phys C. 2007;18(04):455-462.

[35] Geier M, Greiner A, Korvink J. A factorized central moment lattice Boltzmann method. The European Physical Journal Special Topics. 2009;171(1):55-61.

[36] Premnath K, Banerjee S. Incorporating forcing terms in cascaded lattice Boltzmann approach by method of central moments. Phys Rev E. 2009;80(3):036702.

[37] Premnath K, Banerjee S. On the three-dimensional central moment lattice Boltzmann method. J Stat Phys. 2011;143(4):747-794.

[38] Geier M, Schönherr M, Pasquali A, et al. The cumulant lattice Boltzmann equation in three dimensions: Theory and validation. Comput Math Appl. 2015;70(4):507-547.

[39] Lycett-Brown D, Luo K. Multiphase cascaded lattice Boltzmann method. Comput Math Appl. 2014;67(2):350-362.

[40] De Rosis A. Nonorthogonal central-moments-based lattice boltzmann scheme in three dimensions. Phys Rev E. 2017;95:013310.

[41] De Rosis A. Non-orthogonal central moments relaxing to a discrete equilibrium: A d2q9 lattice boltzmann model. Europhys Lett. 2017;116(4):44003.

[42] Mininni P, Pouquet A, Montgomery D. Small-scale structures in three-dimensional magnetohydrodynamic turbulence. Phys Rev Lett. 2006;97(24):244503.

[43] Alexakis A, Mininni PD, Pouquet A. Shell-to-shell energy transfer in magnetohydrodynamics. i. steady state turbulence. Phys Rev E. 2005 Oct;72:046301. Available from: http://link.aps.org/doi/10.1103/PhysRevE.72.046301.

[44] Politano H, Pouquet A, Sulem PL. Inertial ranges and resistive instabilities in two-dimensional magnetohydrodynamic turbulence. Phys Fluids B. 1989;1(12):2330-2339.

[45] Pouquet A. On two-dimensional magnetohydrodynamic turbulence. J Fluid Mech. 1978; 88(1):1-16.

[46] Montessori A, Prestininzi P, Rocca ML, et al. Lattice boltzmann approach for complex non-equilibrium flows. Phys Rev E. 2015;92.

[47] Lai H, Xu A, Zhang G, et al. Nonequilibrium thermodynamic effects on the rayleigh-taylor instability in compressible flows. Phys Rev E. 2016;94.

[48] Lin C, Luo KH, Fei L, et al. A multi-component discrete boltzmann model for nonequilibirum reactive flows. Scientific Reports. 2017;7. 